\title{
Prevalence of human papillomavirus infection among Iranian women using COBAS HPV DNA testing
}

\author{
Farzane Jamdar ${ }^{1}$, Farah Farzaneh ${ }^{2 *}$ D, Fariba Navidpour ${ }^{3}$, Sarang Younesi ${ }^{4}$, Payam Balvayeh ${ }^{5}$, \\ Maryamsadat Hosseini ${ }^{1}$ and Robabeh Ghodssi-Ghasemabadi ${ }^{6}$
}

\begin{abstract}
Background: Persistent infection with High Risk Human Papillomavirus (HR HPV) types plays a major role in the development of cervical cancer. Therefore, the detection of HR HPV types is an essential part of cervical cancer screening. The aim of this study was to estimate the prevalence of HR HPV infection among healthy women undergoing routine cervical cancer screening in Iran.
\end{abstract}

Methods: In this cross-sectional study, the results of HPV DNA typing in 2453 normal Iranian women who were referred for routine cervical cancer screening from September 2015 to March 2017 were analyzed. Participants were screened using COBAS assay for HPV DNA typing and liquid based cytology.

Results: A total of 2453 healthy sexually active women were included in this study. The mean age was $35.1 \pm 8$. 08 years. The overall prevalence of HR HPV infection was 10.3\%. HPV16 was found in 73 (3\%) women. The prevalence of HPV18 and other HR HPV types were 16(0.7\%) and166 (8.2\%), respectively. Approximately, 5\% of the study population had an abnormal cervical cytology (ASCUS or worse), of whom 34\% were infected by HR HPV.

Conclusion: The prevalence of HR HPV infection among Iranian women has increased in the recent years which indicates the need for public education and health planning to prevent this cancer through vaccination and early diagnosis using screening tests. HPV DNA typing, diagnosis and the distribution of prevalent genotypes should be considered in the development of comprehensive cervical cancer prevention programs in Iran.

\section{Background}

Cervical cancer is the third most common gynecologic cancer and the fourth leading cause of cancer death among women worldwide [1]. It has been demonstrated that persistent infection with high risk human papilloma virus (HR HPV) types plays a major role in the development of cervical intraepithelial neoplasia and cervical cancer [2-6]. Depending on the prevalence of HPV types in cervical cancer and its precursors, HPV types are classified as "low risk" and "high risk" [7]. Although HPV infection is the most common sexually transmitted disease in the world, the most detected HPV infections are transient and not expected to cause future high-grade cervical disease: $54 \%$ resolve spontaneously in one

\footnotetext{
* Correspondence: jamdar.farzane@gmail.com

${ }^{2}$ Head of the preventive Gynecology Research Center (PGRC) Shahid

Beheshti university of Medical science, Imam Hossein Hospital, Tehran, Iran

Full list of author information is available at the end of the article
}

year and $91 \%$ in two years $[8,9]$. Practically the prevalence and type-distribution of HPV infection vary between populations in different countries [10].

In the study by Khodakarami et al. 2012, the prevalence of high-risk HPV infection in healthy women was $5.1 \%$ in Tehran, Iran and the HPV 16 was the most common detected virus genotype (17). HPV 16 has been the most common type of infection in previous studies [11-17]. In the recent years, HPVs 31, 33, 45 and 58 have mostly been observed in many countries in East Asia [18]. HPV vaccination has been proven to offer near $100 \%$ protection against the development of cervical cancer and pre-cancerous lesion of HPV among individuals who have not previously been infected with HPV [11]. Based on these results, HPV DNA typing in clinical setting and the identification of HR-HPV genotypes is an important part of cervical cancer screening, as well as a means of providing valuable evidences necessary for the 
prevention and management of this cancer [11]. HPV DNA typing is not considered as routine practices of cervical cancer screening in Iran yet. The aim of this study was to estimate the prevalence of HR HPV infection among Iranian healthy women undergoing routine cervical cancer screening using COBAS HPV typing assay. The results of the current study might help us in making public health decisions regarding the cervical cancer screening and HPV vaccination to prevent and decrease the incidence of this cancer in Iran.

\section{Methods}

\section{Study population}

In this cross-sectional study, the result of the HPV screening of 2453 healthy women who were referred to Nilou laboratory, Tehran, Iran for cervical cancer routine screening from September 2015 to March 2017 were analyzed. Demographic data, cervical cytology reports, and HPV test results were collected. Demographic data were recorded by trained laboratory staff. Cervical smears were obtained from the cervix of each participant with cyto-brush for liquid-based cytological analysis and HPV DNA testing by gynecologists. Cervical cytology smears were read by acyto pathologist who was unaware of participants' HPV DNA test results. After providing HPV DNA test and cytology results, women with ASCUS or worse or positive HR HPV DNA were referred to a gyneconcologist for colposcopy examination.

\section{HPV genotyping}

Roche cobas ${ }^{\ominus} \mathrm{HPV}$ test (Roche Molecular Systems, Pleasanton CA), which is one of four approved HPV tests by the US Food and Drug Administration was used for HPV DNA typing. The $\mathrm{COBAS}^{\oplus} \mathrm{HPV}$ test can detect 14 high risk HPVs. This HPV DNA testing method separately detects HPV16 and HPV18and a pool of 12 other high-risk (HR) HPVs types together $(31,33,35,39,45,51,52,56,58,59,66,68)$ $[19,20]$.

After obtaining smeared cell slides for ThinPrep liquid-based cytology test, the remaining cell samples on the cytobrush were stored at room temperature for further analysis (HPV DNA is stable for 4 months). The HPV detection and pathological diagnosis were performed independently in two departments. Roche $\mathrm{COBAS}^{\ominus} \mathrm{HPV}$ test is an automated qualitative in vitro test for the detection of human papilloma virus (HPV) DNA in patient specimens. The test utilizes amplification of target DNA by the polymerase Chain reaction (PCR) and nucleic acid hybridization for detection of 14 high-risk HPV (hr-HPV) types in a single analysis. The test specifically identifies HPV16 and HPV18 while concurrently detecting the rest of the high risk types $(31,33,35,39,45,51,52,56,58,59,66$, and 68$)$ at clinically relevant infection levels.

\section{Statistical analysis}

Continuous data were represented by mean and standard deviation, count and proportion was applied to show categorical data. The distribution of categorical data in different groups was tested using chi-squared test. Proportions were reported with 95\% confidence interval. The trends of proportions were tested using Cochrane-Armitage test. The significance level was set at 0.05 level and SPSS software version 21 was used for analysis.

The study was approved by the Preventative Gynecology Research Center (PGRC), Shahid Beheshti University of Medical Sciences ethics committee. All of the participants were informed about the study and signed the written consent form.

\section{Results}

A total of 2453 healthy sexually active women were included in this study. The mean age was $35.1 \pm 8.08$ years. The overall prevalence of HR HPV infection was $10.3 \%$ (253 women).

HPV16 infection (alone or with other HR HPVs) was detected in 73 (3\%) women. HPV18 infection (alone or with other HR HPV) was detected in $0.7 \%$ of participants, other 12 HR HPV types $(31,33,35,39,45,51,52,56,58,59,66,68)$ were detected in $8.2 \%$ of women according to COBAS HPV DNA assay. The prevalence of HPV genotype is summarized in Table 1 . There was a statistically significant decrease in the HPV prevalence with age categories $(P<0.001)$. Results are shown in Fig. 1 .

Cervical cytology results of 793 women were available. The mean age of women with known and unknown cervical cytology results were $34.37 \pm 7.51$ and $35.44 \pm 8.31$, respectively.

Majority of them with known cervical cytology results [95\% (753 out of 793)] had normal pap-smear. The prevalence of atypical squamous cells of undetermined significance (ASCUS), low-grade squamous intraepithelial lesion (LSIL), high-grade squamous intraepithelial lesion (HSIL), and atypical squamous cells cannot exclude high grade SIL (ASC-H) were (3\%), (1.9\%), (0\%), and $(0.1 \%)$, respectively in this population.HR HPV DNA was detected in $13.5 \%$ (102 out of 753) of women with normal cytology. The prevalence of HR HPV was significantly higher in patient with abnormal cervical cytology than women with normal cervical cytology (34\% versus $13.3 \%, P<0.001)$. The prevalence of HR HPV infection increased with greater diagnosis severity. The prevalence of HR HPV infection was $54.2 \%$ in ASCUS and $66.7 \%$ in LSIL. The results of cervical cytology are presented in Table 2 for positive and negative findings of HPV. Other $12 \mathrm{HR}$ HPV types were predominant types of infection in all grades of both abnormal and normal cytology. The distribution HPV genotype are shown in Table 3 based on cytology results. 
Table 1 HPV genotype results

\begin{tabular}{llll}
\hline HPV TYPING & Frequency & Prevalence & $95 \%$ Cl for Prevalence \\
\hline HPV 16 (single infection) & $44(1.8)$ & $1.8 \%$ & $(1.3 \%-2.4 \%)$ \\
HPV 18 (single infection) & $10(0.4)$ & $0.4 \%$ & $(0.21 \%-0.78 \%)$ \\
HPV 16\& HPV18 as co-infections & 0 & $0 \%$ & - \\
Other HR HPVs (31,33,35,39,45,51,52,56,58,59,66,68) & $166(6.8)$ & $6.8 \%$ & $(5.8 \%-7.9 \%)$ \\
Other HR + 16 & $27(1.1)$ & $1.1 \%$ & $(0.74 \%-1.6 \%)$ \\
Other HR + 18 & $4(0.2)$ & $0.2 \%$ & $(0.05 \%-0.45 \%)$ \\
Other HR + 16+18 & $2(0.1)$ & $0.1 \%$ & $(0.01 \%-0.33 \%)$ \\
Sum* & 253 of $2453(10.3)$ & $10.3 \%$ & $(9.2 \%-11.6 \%)$ \\
Total & $2453(100)$ & $100 \%$ & - \\
\hline
\end{tabular}

*Sum of the HPV typing

HPV: Human Papilloma Virus, HR: High Risk

\section{Discussion}

In this study the overall prevalence of HR HPV infection was $10.3 \%$ using COBAS HPV DNA typing assay among healthy women subjected to routine cervical cancer screening. Approximately, 5\% of available cervical cytology results were abnormal (ASCUS or worse) the prevalence of HPV infection decreased significantly with age. The highest prevalence among individuals under the age of 25 was $15.6 \%$, which gradually decreased and reached $4.5 \%$ among women over the age of 44 years. Zandi et al. (2008) reported that the prevalence of HPV infection in women undergoing routine pap smears was 5.5\% in Bushehr, Iran [21]. Moreover, Khodakarami et al. (2012) reported the $7.8 \%$ HPV infection prevalence in Tehran using GP5+/6+ PCR-based assay, but in their report, the prevalence of HR HPV infection was $5.1 \%$ with no significant variation by age [17]. The prevalence of abnormal papsmear was $4.1 \%$ in their study and HPV16 was the most prevalent genotype [17]. The study of Khodakarami et al. (2012) on 18- to 59-year-old women in Tehran showed that the prevalence of HPV infection was significantly higher among women in polygamous marriages, divorced women, and those reporting husband's absence from home for more than 7 nights/ month [17]. In another study by Yousefzadeh et al. (2013), the prevalence of HPV infection in women was 31.1\%in Tehran, Iran. The reason for this rate of HPV prevalence in this study was that they examined the prevalence of low-risk and high-risk HPVs together and the studied population was not general population, but were those who were examined for gynecological symptoms [22]. In this study, we used COBAS method to detect HPV infection, which is approved by FDA. The approval is based on ATHENA study which was performed on more than 47,000 women in the US [23]. Based on the ATHENA study, COBAS is the only FDA approved method for primary cervical cancer screening $[23,24]$. In the ATHENA study, it was found that primary screening of HPV in women $\geq 25$ years is as effective as dual screening in conjunction with cytology, but initial screening with HPV alone requires less testing [24].

The prevalence of HPV infection and its distribution differed by age, geographic region, and cytology findings [11]. Wu EQ et al. (2013), and Zhao (2015) reported



Fig. $1 \mathrm{HPV}$ prevalence based on age categories 
Table 2 Prevalence of HPV based on cytology

\begin{tabular}{|c|c|c|c|}
\hline \multirow[b]{2}{*}{ PAP smear report } & \multirow[b]{2}{*}{$N(\%)$} & \multicolumn{2}{|l|}{ HPV } \\
\hline & & $\begin{array}{l}\text { Negative } \\
\mathrm{N}(\%)\end{array}$ & $\begin{array}{l}\text { Positive } \\
N(\%)\end{array}$ \\
\hline Normal & $753(95)$ & $651(86.5)$ & $102(13.5)$ \\
\hline ASCUS & $24(3)$ & $11(45.8)$ & $13(54.2)$ \\
\hline LSIL & $15(1.9)$ & $5(33.3)$ & $10(66.6)$ \\
\hline $\mathrm{HSIL}$ & $0(0)$ & 0 & 0 \\
\hline $\mathrm{ASCH}$ & $1(0.1)$ & $1(100)$ & 0 \\
\hline Total & 793 (100) & $668(84.2)$ & $125(15.8)$ \\
\hline
\end{tabular}

ASCUS: Atypical squamous cells of undetermined significance, LSIL: Low-Grade Squamous Intraepithelial Lesion, HSIL: High-Grade Squamous Intraepithelial Lesion, ASCH: A typical squamous cells cannot excluded high grade SIL

$14.3 \%$ and $15.5 \%$, respectively for the prevalence of HPV infection among Chinese women [11, 12]. Wu et al. (2017) reported $22.7 \%$ and $17.3 \%$ for the prevalence of any HPV infections and oncogenic HPV type, respectively in China [25].

The prevalence of HPV infection was 19\% among Portuguese women in (2011) [26]. Furthermore, the prevalence of high risk HPV was reported $8 \%$ in Italy by Giorgi et al. (2012) [15]. While, based on a study by Monsonegoet et al. (2012), on women in Paris, the overall prevalence of HPV infection differed using APTIMA HPV assay (AHPV) (10.1\%) and Hybrid capturer 2 (HC2)(15\%)assay [14]. McQuillan, et al. (2017) reported that the prevalence of $39.9 \%$ for any and $20.4 \%$ for high risk HPVs among American women aged 18-59 years [27].

The prevalence of HR HPV infection among Iranian women was more than Italian women but it was less than American, Chinese and European (Paris and Portugal) records. Compared with khodakarami et al. 2012, and Zandi et al. 2008, we have been confronting a rising rate of HPV infection in our country since 2008. It should be emphasized that the HPV DNA detection was carried out using different methods in these studies. Moreover, in our study, the rate of abnormal pap-smear (5\%) increased in comparison with the rate (4.1\%) reported by khodakarami et al. 2012 .

An increase in the prevalence of HPV infection in recent years has multiple causes. Iran's population is predominantly young, and we are witnessing a change in the sexual behavior of teenagers compared to the past. These behavioral changes and lack of adequate education about sexual issues have led to a high rate of referral to genecology clinics due to genital wart and the complications of HPV infection. Another cause of increased prevalence of HPV is the prevalent use of hookah among teenagers and young people. This type of tobacco has traditionally been used in Iran. Also, the community is familiar with the dangers and disadvantages of smoking, but considering the history of smoking hookah in Iran, most people consider this as a safe form of tobacco and are not aware of its dangers.

On the other hand, vaccination against HPV infection is not currently routine in Iran and the cost of the vaccine is not covered by the insurance companies either. Also, the study of Jalilvand et al. (2014) showed that in addition to cervical cancers, HPV type 16 and 18 are also the most common types of viruses in other HPV- induced cancers (such as head and neck SSCs). Based on this, the existing vaccines, in addition to reducing the cervical cancer, lead to decreased in other HPV-dependent cancers [28]. Providing the statistics on the high-risk HPV prevalence in Iran will assist us in designing accurate health educational programs to reduce the prevalence of HPV infection and cervical cancer and to diagnose the disease in early stages in our country in the coming years.

The limitation of the current study are as follows:

First, the COBAS HPV testing is organized for clinical decision making in order to manage cervical lesions. This method reports HPV16, 18, and 12 other high risk types of HR HPV together. Therefore, we were not able to separately find out the prevalence of other 12 types of HR HPV. While, knowing the common genotypes of HPV infection has clinical significance for vaccination. Second, the HPV analysis is carried out at NILOU laboratory using the COBAS HPV DNA testing and many laboratories from different cities of Iran analyzed cytological specimens themselves and just referred the samples to NILOU laboratory for HPV typing using the COBAS assay. Therefore, pap-smear results of many patients were not available.

\section{Conclusion}

The results of the current study showed that the prevalence of HPV infection in Iran is close to the worldwide HPV prevalence and it might increase. Therefore, all suggested preventative issues (education on sexual activity, social behavior, vaccination and screening programs) by

Table 3 Distribution of HPV genotype based on cytology results

\begin{tabular}{llllllll}
\hline & HPV 16 & HPV 18 & HPV HR & $16+$ HR & $16+18+$ HR & $18+16$ & $18+$ HR \\
\hline Normal & $17(16.7 \%)$ & $2(2 \%)$ & $71(69.5 \%)$ & $11(10.8)$ & $11 \%$ & 0 & 0 \\
ASCUS & $2(15.4 \%)$ & 0 & $8(61.5 \%)$ & $3(23.1 \%)$ & 0 & 0 & 0 \\
LSIL & $3(30 \%)$ & 0 & $5(50 \%)$ & $1(10 \%)$ & 0 & $1(10 \%)$ \\
\hline
\end{tabular}

ASCUS: Atypical squamous cells of undetermined significance, LSIL: Low-Grade Squamous Intraepithelial Lesion, HSIL: High-Grade Squamous Intraepithelial Lesion, ASCH: Atypicalsquamouscells cannot excluded high grade SILI 
international health organizations (i.e. WHO) regarding HR HPV could be considered for Iranian population by health workers. The present study is the first study in Iran to investigate the prevalence of HPV using the COBAS method.

\section{Acknowledgments}

The authors would like to thank Dr Taheri MM, the NILOU medical laboratory manager and their personnel for their contribution to this study. Special thanks to thelaboratory staff, Mrs. Navidpour, for collecting the data.

\section{Funding}

This study was funded by Preventive Gynecology Research Center (PGRC) of Shahid Beheshti University of Medical science.

\section{Availability of data and materials}

Authors can confirm that all relevant data are included in the article and materials are available on request from the authors.

\section{Authors' contributions}

FJ designed the study and wrote the manuscript. FF initiated and designed the study and concept, and edited the final version of the manuscript; FN collected the data for analysis; SY helped to collect the data and conducted HPV assay; PB conducted pop-smear test and helped to collect the data; MH assisted in the development of the concepts; RGG conducted statistical analysis and data interpretation. All authors read and approved the final manuscript.

\section{Ethics approval and consent to participate}

The study was approved by the preventative Gynecology Research Center (PGRC), Shahid Beheshti University of Medical Sciences ethics committees. All study participants were informed about the study and signed the written consent form.

\section{Consent for publication}

Not applicable.

\section{Competing interests}

The authors declare that they have no competing interests.

\section{Publisher's Note}

Springer Nature remains neutral with regard to jurisdictional claims in published maps and institutional affiliations.

\section{Author details}

'Preventive Gynecology Research Center (PGRC) Shahid Beheshti university of Medical science, Imam Hossein Hospital, Tehran, Iran. ${ }^{2}$ Head of the preventive Gynecology Research Center (PGRC) Shahid Beheshti university of Medical science, Imam Hossein Hospital, Tehran, Iran. ${ }^{3}$ Manager of post analytical quality control department in Nilou lab, Tehran, Iran. ${ }^{4}$ Technical manager of nilou laboratory, Tehran, Iran. ${ }^{5}$ Anatomical and clinical pathologist, Tehran, Iran. ${ }^{6}$ Department of Biostatistics, Faculty of Medical Sciences, Tarbiat Modares University, Tehran, Iran.

Received: 12 September 2017 Accepted: 16 January 2018 Published online: 25 January 2018

\section{References}

1. Farzaneh F, et al. Application of tumor markers SCC-ag, CEA, and TPA in patients with cervical precancerous lesions. Asian Pac J Cancer Prev. 2014; 15(9):3911-4.

2. Zur Hausen H. Papillomaviruses and cancer: from basic studies to clinical application. Nat Rev Cancer. 2002;2(5):342.

3. Bosch F, et al. The causal relation between human papillomavirus and cervical cancer. J Clin Pathol. 2002;55(4):244-65.

4. Burd EM. Human papillomavirus and cervical cancer. Clin Microbiol Rev. 2003;16(1):1-17.

5. Cuzick J, et al. Management of women who test positive for high-risk types of human papillomavirus: the HART study. Lancet. 2003;362(9399):1871-6.
6. Koutsky LA, et al. A cohort study of the risk of cervical intraepithelial neoplasia grade 2 or 3 in relation to papillomavirus infection. N Engl J Med. 1992;327(18):1272-8.

7. Wu D, et al. Prevalence of genital human papillomavirus infection and genotypes among women from Fujian province, PR China. European Journal of Obstetrics \& Gynecology and Reproductive Biology. 2010;151(1): 86-90.

8. Cobo F, Concha Á, Ortiz M. Human papillomavirus (HPV) type distribution in females with abnormal cervical cytology. A correlation with histological study. The open virology journal. 2009;3:60.

9. Safaeian $\mathrm{M}$, et al. Risk of precancer and follow-up management strategies for women with human papillomavirus-negative atypical squamous cells of undetermined significance. Obstet Gynecol. 2007;109(6):1325-31.

10. Smith JS, et al. Human papillomavirus type distribution in invasive cervical cancer and high-grade cervical lesions: a meta-analysis update. Int J Cancer. 2007;121(3):621-32.

11. Wu E-Q, et al. Prevalence of type-specific human papillomavirus and pap results in Chinese women: a multi-center, population-based cross-sectional study. Cancer Causes Control. 2013;24(4):795-803.

12. Zhao $\mathrm{Y}$, et al. Multi-center cross-sectional study on type-specific human papillomavirus infection among Chinese women. Zhonghua liu xing bing xue za zhi=. Zhonghua liuxingbingxue zazhi. 2015;36(12):1351-6.

13. Sjoeborg KD, et al. HPV genotype distribution according to severity of cervical neoplasia. Gynecol Oncol. 2010;118(1):29-34.

14. Monsonego J, et al. Prevalence of type-specific human papillomavirus infection among women in France: implications for screening, vaccination, and a future generation of multivalent HPV vaccines. Vaccine. 2012;30(35): $5215-21$.

15. Giorgi RP, et al. Human papilloma virus (HPV), cervical cancer incidence and screening uptake: differences among northern, central and southern Italy. Epidemiol Prev. 2012;36(2):108-19.

16. Lin $\mathrm{H}$, et al. High prevalence of genital human papillomavirus type 52 and 58 infection in women attending gynecologic practitioners in South Taiwan. Gynecol Oncol. 2006;101(1):40-5.

17. Khodakarami $\mathrm{N}$, et al. Human papillomavirus infection in women with and without cervical cancer in Tehran, Iran. Int J Cancer. 2012;131:156-61.

18. Li N, et al. Human papillomavirus type distribution in 30,848 invasive cervical cancers worldwide: variation by geographical region, histological type and year of publication. Int J Cancer. 2011;128(4):927-35.

19. Isidean SD, Coutlée F, Franco EL. Cobas ${ }^{\oplus} 4800$ HPV test, a real-time polymerase chain reaction assay for the detection of human papillomavirus in cervical specimens. Expert Rev Mol Diagn. 2014;14(1):5-16.

20. Schiffman $M$, et al. The role of human papillomavirus genotyping in cervical cancer screening: a large-scale evaluation of the cobas HPV test. Cancer Epidemiology and Prevention Biomarkers. 2015;24(9):1304-10.

21. Zandi K, et al. Prevalence of various human papillomavirus (HPV) genotypes among women who subjected to routine pap smear test in Bushehr city (south west of Iran) 2008-2009. Virol J. 2010;7(1):65.

22. Yousefzadeh A, et al. Human papillomavirus (HPV) prevalence and types among women attending regular gynecological visit in Tehran. Iran Clin Lab. 2014;60(2):267-73.

23. Wright TC, et al. The ATHENA humuan papilomavirus study : design, methods, and baseline results. Am J Obstst Gynecol. 2012;206(1):46. -e1-46-e11

24. wright TC, et al. Primary cervical cancer screening with humuan papilomavirus:end of study results from the ATHENA study using HPV as the first-line screening test. Am J Obstst Gynecol. 2015;136:189-97.

25. Wu, X., et al., Prevalence of type-specific human papillomavirus infection among 18-45 year-old women from the general population in Liuzhou, Guangxi Zhuang Autonomous Region: a cross-sectional study. Zhonghua liu xing bing xue za zhi= Zhonghua liuxingbingxue zazhi, 2017. 38(4): p. 467.

26. Pista $A$, et al. Prevalence of human papillomavirus infection in women in Portugal: the CLEOPATRE Portugal study. Int J Gynecol Cancer. 2011;21(6): $1150-8$.

27. McQuillan G, et al. Prevalence of HPV in Adults Aged 18-69: United States, 2011-2014. NCHS data brief. 2017;280:1.

28. Jalilvand S, Shoja Z, Hamkar R. Human papillomavirus burden in different cancers in Iran: a systematic assessment. Asian Pac J Cancer Prev. 2014; 15(17):7029-35. 